\title{
A Note on Certain Laplace Transforms of Convolution-Type Integrals Involving Product of Two Generalized Hypergeometric Functions
}

\author{
Arjun Kumar Rathie, ${ }^{1}$ Young Hee Geum, ${ }^{2}$ and Hwajoon Kim ${ }^{3}{ }^{3}$ \\ ${ }^{1}$ Department of Mathematics, Vedant College of Engineering and Technology, Rajasthan Technical University, \\ Bundi 323021, India \\ ${ }^{2}$ Dankook University, Cheonan, Republic of Korea \\ ${ }^{3}$ Kyungdong University, Yangju, Republic of Korea \\ Correspondence should be addressed to Hwajoon Kim; cellmath@gmail.com
}

Received 30 September 2020; Accepted 22 May 2021; Published 30 May 2021

Academic Editor: Xue-Bo Chen

Copyright (c) 2021 Arjun Kumar Rathie et al. This is an open access article distributed under the Creative Commons Attribution License, which permits unrestricted use, distribution, and reproduction in any medium, provided the original work is properly cited.

The aim of this research paper is to provide as many as forty-five attractive Laplace transforms of convolution type related to the product of generalized hypergeometric functions. These are achieved by employing summation theorems for the series ${ }_{p} F_{p-1}$ (for $p=2,3,4$, and 5) available in the literature. The obtained research result is to provide an easier method than the existing method.

\section{Introduction and Results' Required}

The theory of hypergeometric and generalized hypergeometric functions [1-3] are fundamental in the field of mathematics, engineering mathematics, and mathematical physics. Most of the commonly used functions that occur in the analysis are special cases or limiting cases of ${ }_{2} F_{1}$ and ${ }_{1} F_{1}$.

It is well known that ${ }_{2} F_{1}$ and ${ }_{1} F_{1}$ defined by

$$
\begin{gathered}
{ }_{2} F_{1}\left[\begin{array}{c}
a, b \\
c
\end{array} \mid z\right]=\sum_{n=0}^{\infty} \frac{(a)_{n}(b)_{n}}{(c)_{n}} \frac{z^{n}}{n !}, \\
{ }_{1} F_{1}\left[\begin{array}{l}
a \\
c
\end{array} \mid z\right]=\sum_{n=0}^{\infty} \frac{(a)_{n}}{(c)_{n}} \frac{z^{n}}{n !},
\end{gathered}
$$

where $(a)_{n}=\Gamma(a+n) / \Gamma(a)$ and $a \neq 0$. Applications related to the detailed content can be found in [4-7].

A natural generalization of this function can be represented by

$$
\begin{aligned}
{ }_{p} F_{q}\left[\begin{array}{c}
(a) \\
(b)
\end{array} \mid z\right] & ={ }_{p} F_{q}\left[\begin{array}{l}
a_{1}, \ldots, a_{p} \\
b_{1}, \ldots, b_{q}
\end{array}\right] z \\
& =\sum_{n=0}^{\infty} \frac{\left(a_{1}\right)_{n} \cdots\left(a_{p}\right)_{n}}{\left(b_{1}\right)_{n} \cdots\left(b_{q}\right)_{n}} \frac{z^{n}}{n !}
\end{aligned}
$$

In the theory of hypergeometric and generalized hypergeometric functions, the following classical summation theorems for the series ${ }_{p} F_{p-1}$ (for $p=2,3,4$, and 5) play an important role [3, 4].

Gauss' summation theorem [8]:

$$
{ }_{2} F_{1}\left[\begin{array}{c}
a, b \\
c
\end{array} \mid 1\right]=\frac{\Gamma(c) \Gamma(c-a-b)}{\Gamma(c-a) \Gamma(c-b)}=\Delta_{1}(a, b, c),
$$

which provided $\Re(c-a-b)>0$.

Gauss's second summation theorem [9]: 


$$
{ }_{2} F_{1}\left[\begin{array}{c|c}
a, b & \frac{1}{2} \\
\frac{1}{2}(a+b+1)
\end{array}\right]=\frac{\Gamma(1 / 2) \Gamma((1 / 2)(a+b+1))}{\Gamma((1 / 2) a+(1 / 2)) \Gamma((1 / 2) b+(1 / 2))}=\Delta_{2}(a, b) .
$$

Bailey's summation theorem [8]:

$$
{ }_{2} F_{1}\left[\begin{array}{c|c}
a, 1-a & 1 \\
b & \frac{1}{2}
\end{array}\right]=\frac{\Gamma((1 / 2) b) \Gamma((1 / 2) b+(1 / 2))}{\Gamma((1 / 2) b+(1 / 2) a) \Gamma((1 / 2) b-(1 / 2) a+(1 / 2))}=\Delta_{3}(a, b) .
$$

Kummer's summation theorem [8]:

Watson summation theorem [8]:

${ }_{2} F_{1}\left[\begin{array}{c}a, b \\ 1+a-b\end{array}\right]=\frac{\Gamma(1+(1 / 2) a) \Gamma(1+a-b)}{\Gamma(1+a) \Gamma(1+(1 / 2) a-b)}=\Delta_{4}(a, b)$.

$$
\begin{aligned}
& { }_{3} F_{2}\left[\begin{array}{c}
a, b, c \\
(1 / 2)(a+b+1), 2 c
\end{array} \mid \begin{array}{c}
1
\end{array}\right] \\
& =\frac{\Gamma(1 / 2) \Gamma(c+(1 / 2)) \Gamma((1 / 2) a+(1 / 2) b+(1 / 2)) \Gamma(c-(1 / 2) a-(1 / 2) b+(1 / 2))}{\Gamma((1 / 2) a+(1 / 2)) \Gamma((1 / 2) b+(1 / 2)) \Gamma(c-(1 / 2) a+(1 / 2)) \Gamma(c-(1 / 2) b+(1 / 2))} \\
& =\Delta_{5}(a, b, c),
\end{aligned}
$$

which provided $\mathfrak{R}(2 c-a-b)>-1$.

Dixon's summation theorem [9]:

$$
\begin{aligned}
& { }_{3} F_{2}\left[\begin{array}{c}
a, b, c \\
1+a-b, 1+a-c
\end{array}\right] \\
& =\frac{\Gamma(1+(1 / 2) a) \Gamma(1+a-b) \Gamma(1+a-c) \Gamma(1+(1 / 2) a-b-c)}{\Gamma(1+a) \Gamma(1+(1 / 2) a-b) \Gamma(1+(1 / 2) a-c) \Gamma(1+a-b-c)} \\
& =\Delta_{6}(a, b, c),
\end{aligned}
$$

which provided $\mathfrak{R}(a-2 b-2 c)>-2$.

Whipple's summation theorem [9]:

$$
\begin{aligned}
& { }_{3} F_{2}\left[\begin{array}{c}
a, 1-a, c \\
e, 1+2 c-e^{\mid}
\end{array}\right] \\
& =\frac{2^{1-2 c} \pi \Gamma(e) \Gamma(1+2 c-e)}{\Gamma((1 / 2) a+(1 / 2) e) \Gamma((1 / 2)-(1 / 2) a+(1 / 2) e) \Gamma(c+(1 / 2) a-(1 / 2) e+(1 / 2)) \Gamma(c-(1 / 2) a-(1 / 2) e+1)} \\
& =\Delta_{7}(a, c, e),
\end{aligned}
$$

which provided $\mathfrak{R}(c)>0$.

Second Whipple's summation theorem [1]: 


$$
\begin{aligned}
& \left.{ }_{4} F_{3}\left[\begin{array}{c}
a, 1+\frac{1}{2} a, b, c \\
\frac{1}{2} a, a-b+1, a-c+1
\end{array}\right]-1\right] \\
& =\frac{\Gamma(a-b+1) \Gamma(a-c+1)}{\Gamma(a+1) \Gamma(a-b-c+1)} \\
& =\Delta_{8}(a, b, c) .
\end{aligned}
$$

Dougall's summation theorem [1]:

$$
\begin{aligned}
& { }_{5} F_{4}\left[\begin{array}{c}
a, 1+\frac{1}{2} a, c, d, e \quad|1| \\
\frac{1}{2} a, a-c+1, a-d+1, a-e+1
\end{array}\right] \\
& =\frac{\Gamma(a-c+1) \Gamma(a-d+1) \Gamma(a-e+1) \Gamma(a-c-d-e+1)}{\Gamma(a+1) \Gamma(a-d-e+1) \Gamma(a-c-e+1) \Gamma(a-c-d+1)} \\
& =\Delta_{9}(a, c, d, e) .
\end{aligned}
$$

In addition to this, we have the following general result of the Laplace transform of convolution-type integrals involving the product of two generalized hypergeometric functions available in the literature, see $[6,7]$ :

$$
\begin{aligned}
& \left.\int_{0}^{\infty} e^{-s t}\left\{\int_{0}^{t} \tau^{\mu-1}(t-\tau)^{\nu-1}{ }_{p} F_{q}\left[\begin{array}{l}
(a) \\
(b)
\end{array}\right]\right]_{p^{\prime}} F_{q^{\prime}}\left[\begin{array}{l}
\left(a^{\prime}\right) \\
\left(b^{\prime}\right)
\end{array} \mid k^{\prime}(t-\tau)\right] \mathrm{d} \tau\right\} \mathrm{d} t \\
& =\Gamma(\mu) \Gamma(v) s^{-\mu-\nu}{ }_{p+1} F_{q}\left[\begin{array}{c}
(a), \mu \\
(b)
\end{array} \frac{k}{s}\right]_{p^{\prime}+1} F_{q^{\prime}}\left[\begin{array}{c}
\left(a^{\prime}\right), v \\
\left(b^{\prime}\right)
\end{array} \mid \frac{k^{\prime}}{s}\right] .
\end{aligned}
$$$$
\text { (1). The }
$$
results obtained earlier by Milovanovic et al. [1,2] follow special cases of our main findings.

\section{A Note on Certain Laplace Transforms of Convolution-Type Integrals Involving Product of Two Generalized Hypergeometric Functions}

In this section, we shall establish in all forty-five Laplace transforms of convolution type related to the product of two generalized hypergeometric functions mentioned in the following theorems. All delta values that appear in this section are shown in Section 1.

Theorem 1. For $\mathfrak{R}(s)>0, \quad \mathfrak{R}(d)>0, \quad \mathfrak{R}\left(d^{\prime}\right)>0$, $\Re(c-a-b)>0$, and $\mathfrak{R}\left(c^{\prime}-a^{\prime}-b^{\prime}\right)>0$, the following result holds true:

$$
\begin{aligned}
& \int_{0}^{\infty} e^{-s t}\left\{\int_{0}^{t} \tau^{d-1}(t-\tau)^{d^{\prime}-1}{ }_{2} F_{2}\left[\begin{array}{l}
a, b \\
c, d
\end{array} \mid{ }^{\prime}\right]_{2} F_{2}\left[\begin{array}{c}
a^{\prime}, b^{\prime} \\
c^{\prime}, d^{\prime}
\end{array} \mid(t-\tau) s\right] \mathrm{d} \tau\right\} \mathrm{d} t \\
& =\Gamma(d) \Gamma\left(d^{\prime}\right) s^{-d-d^{\prime}} \Delta_{1}(a, b, c) \Delta_{1}\left(a^{\prime}, b^{\prime}, c^{\prime}\right) .
\end{aligned}
$$

Theorem 2. For $\mathfrak{R}(s)>0, \quad \mathfrak{R}(d)>0, \quad \mathfrak{R}\left(d^{\prime}\right)>0$, and $\Re(c-a-b)>0$, the following result holds true:

$$
\begin{aligned}
& \int_{0}^{\infty} e^{-s t}\left\{\int_{0}^{t} \tau^{d-1}(t-\tau)^{d^{\prime}-1}{ }_{2} F_{2}\left[\begin{array}{l}
a, b \\
c, d
\end{array} \mid \tau s\right] \times{ }_{2} F_{2}\left[\begin{array}{c}
a^{\prime}, b^{\prime} \\
\frac{1}{2}\left(a^{\prime}+b^{\prime}+1\right), d^{\prime}
\end{array} \mid \frac{1}{2}(t-\tau) s\right] \mathrm{d} \tau\right\} \mathrm{d} t \\
& =\Gamma(d) \Gamma\left(d^{\prime}\right) s^{-b-b^{\prime}} \Delta_{1}(a, b, c) \Delta_{2}\left(a^{\prime}, b^{\prime}\right) .
\end{aligned}
$$

Theorem 3. For $\mathfrak{R}(s)>0, \quad \mathfrak{R}(d)>0, \quad \mathfrak{R}\left(d^{\prime}\right)>0$, and $\Re(c-a-b)>0$, the following result holds true:

$$
\begin{aligned}
& \int_{0}^{\infty} e^{-s t}\left\{\int_{0}^{t} \tau^{d-1}(t-\tau)^{d^{\prime}-1}{ }_{2} F_{2}\left[\begin{array}{l}
a, b \\
c, d
\end{array} \mid \tau s\right] \times{ }_{2} F_{2}\left[\begin{array}{c}
a^{\prime}, 1-a^{\prime} \\
b^{\prime}, d^{\prime}
\end{array} \mid \frac{1}{2}(t-\tau) s\right] \mathrm{d} \tau\right\} \mathrm{d} t \\
& =\Gamma(d) \Gamma\left(d^{\prime}\right) s^{-d-d^{\prime}} \Delta_{1}(a, b, c) \Delta_{3}\left(a^{\prime}, b^{\prime}\right) .
\end{aligned}
$$


Theorem 4. For $\mathfrak{R}(s)>0, \quad \mathfrak{R}(d)>0, \quad \mathfrak{R}\left(d^{\prime}\right)>0$, and $\Re(c-a-b)>0$, the following result holds true:

$$
\begin{aligned}
& \int_{0}^{\infty} e^{-s t}\left\{\int_{0}^{t} \tau^{d-1}(t-\tau)^{d^{\prime}-1}{ }_{2} F_{2}\left[\begin{array}{l}
a, b \\
c, d
\end{array} \mid \tau s\right] \times{ }_{2} F_{2}\left[\begin{array}{c}
a^{\prime}, b^{\prime} \\
1+a^{\prime}-b^{\prime}, d^{\prime}
\end{array} \mid(t-\tau) s\right] \mathrm{d} \tau\right\} \mathrm{d} t \\
& =\Gamma(d) \Gamma\left(d^{\prime}\right) s^{-d-d^{\prime}} \Delta_{1}(a, b, c) \Delta_{4}\left(a^{\prime}, b^{\prime}\right) .
\end{aligned}
$$

Theorem 5. For $\mathfrak{R}(s)>0, \quad \mathfrak{R}(d)>0, \quad \mathfrak{R}\left(d^{\prime}\right)>0$, $\mathfrak{R}(c-a-b)>0$, and $\mathfrak{R}\left(2 c^{\prime}-a^{\prime}-b^{\prime}\right)>-1$, the following result holds true:

$$
\begin{aligned}
& \int_{0}^{\infty} e^{-s t}\left\{\int_{0}^{t} \tau^{d-1}(t-\tau)^{d^{\prime}-1}{ }_{2} F_{2}\left[{ }_{c, d}^{a, b} \mid \tau s\right] \times{ }_{3} F_{3}\left[\begin{array}{c}
a^{\prime}, b^{\prime}, c^{\prime} \\
\frac{1}{2}\left(a^{\prime}+b^{\prime}+1\right), 2 c^{\prime}, d^{\prime}
\end{array} \mid(t-\tau) s\right] \mathrm{d} \tau\right\} \mathrm{d} t \\
& =\frac{\Gamma(d) \Gamma\left(d^{\prime}\right)}{s^{d+d^{\prime}}} \Delta_{1}(a, b, c) \Delta_{5}\left(a^{\prime}, b^{\prime}, c^{\prime}\right) .
\end{aligned}
$$

Theorem 6. For $\mathfrak{R}(s)>0, \quad \mathfrak{R}(d)>0, \quad \mathfrak{R}\left(d^{\prime}\right)>0$, $\mathfrak{R}(c-a-b)>0$, and $\mathfrak{R}\left(a^{\prime}-2 b^{\prime}-2 c^{\prime}\right)>-2$, the following result holds true:

$$
\begin{aligned}
& \int_{0}^{\infty} e^{-s t}\left\{\int_{0}^{t} \tau^{d-1}(t-\tau)^{d^{\prime}-1}{ }_{2} F_{2}\left[\begin{array}{l}
a, b \\
c, d
\end{array} \mid \tau s\right] \times{ }_{3} F_{3}\left[\begin{array}{c}
a^{\prime}, b^{\prime}, c^{\prime} \\
1+a^{\prime}-b^{\prime}, 1+a^{\prime}-c^{\prime}, d^{\prime}
\end{array} \mid(t-\tau) s\right] \mathrm{d} \tau\right\} \mathrm{d} t \\
& =\frac{\Gamma(d) \Gamma\left(d^{\prime}\right)}{s^{d+d^{\prime}}} \Delta_{1}(a, b, c) \Delta_{6}\left(a^{\prime}, b^{\prime}, c^{\prime}\right) .
\end{aligned}
$$

Theorem 7. For $\mathfrak{R}(s)>0, \quad \mathfrak{R}(d)>0, \quad \mathfrak{R}\left(d^{\prime}\right)>0$, and $\mathfrak{R}(c-a-b)>0$, the following result holds true:

$$
\begin{aligned}
& \int_{0}^{\infty} e^{-s t}\left\{\int_{0}^{t} \tau^{d-1}(t-\tau)^{d^{\prime}-1}{ }_{2} F_{2}\left[\begin{array}{l}
a, b \\
c, d
\end{array} \mid \tau s\right] \times{ }_{3} F_{3}\left[\begin{array}{c}
a^{\prime}, 1-a^{\prime}, c^{\prime} \\
e^{\prime}, 1+2 c^{\prime}-e^{\prime}, d^{\prime}
\end{array} \mid(t-\tau) s\right] \mathrm{d} \tau\right\} \mathrm{d} t \\
& =\frac{\Gamma(d) \Gamma\left(d^{\prime}\right)}{s^{d+d^{\prime}}} \Delta_{1}(a, b, c) \Delta_{7}\left(a^{\prime}, c^{\prime}, e^{\prime}\right) .
\end{aligned}
$$

Theorem 8. For $\mathfrak{R}(s)>0, \quad \mathfrak{R}(d)>0, \quad \mathfrak{R}\left(d^{\prime}\right)>0$, and $\mathfrak{R}(c-a-b)>0$, the following result holds true: 


$$
\begin{aligned}
& \left.\int_{0}^{\infty} e^{-s t}\left\{\int_{0}^{t} \tau^{d-1}(t-\tau)^{d^{\prime}-1}{ }_{2} F_{2}\left[{ }_{c, d}^{a, b} \mid \tau s\right] \times{ }_{4} F_{4}\left[\begin{array}{c}
a^{\prime}, 1+\frac{1}{2} a^{\prime}, b^{\prime}, c^{\prime} \\
\frac{1}{2} a^{\prime}, a^{\prime}-b^{\prime}+1, a^{\prime}-c^{\prime}+1, d^{\prime}
\end{array}\right]-(t-\tau) s\right] \mathrm{d} \tau\right\} \mathrm{d} t \\
& =\frac{\Gamma(d) \Gamma\left(d^{\prime}\right)}{s^{d+d^{\prime}}} \Delta_{1}(a, b, c) \Delta_{8}\left(a^{\prime}, b^{\prime}, c^{\prime}\right) .
\end{aligned}
$$

Theorem 9. For $\mathfrak{R}(s)>0, \quad \mathfrak{R}(d)>0, \quad \mathfrak{R}\left(f^{\prime}\right)>0$, $\Re(c-a-b)>0$, and $\mathfrak{R}\left(a^{\prime}-c^{\prime}-d^{\prime}-e^{\prime}+1\right)>0$, the following result holds true:

$$
\begin{aligned}
& \left.\int_{0}^{\infty} e^{-s t}\left\{\int_{0}^{t} \tau^{d-1}(t-\tau)^{f^{\prime}-1}{ }_{2} F_{2}\left[{ }_{c, d}^{a, b} \mid \tau s\right] \times{ }_{5} F_{5}\left[\begin{array}{c}
a^{\prime}, 1+\frac{1}{2} a^{\prime}, c^{\prime}, d^{\prime}, e^{\prime} \\
\frac{1}{2} a^{\prime}, a^{\prime}-c^{\prime}+1, a^{\prime}-d^{\prime}+1, a^{\prime}-e^{\prime}+1, f^{\prime}
\end{array}\right](t-\tau) s\right] \mathrm{d} \tau\right\} \mathrm{d} t \\
& =\frac{\Gamma(d) \Gamma\left(f^{\prime}\right)}{s^{d+f^{\prime}} \Delta_{1}(a, b, c) \Delta_{9}\left(a^{\prime}, c^{\prime}, d^{\prime}, e^{\prime}\right) .}
\end{aligned}
$$

Theorem 10. For $\mathfrak{R}(s)>0, \mathfrak{R}(d)>0$, and $\mathfrak{R}\left(d^{\prime}\right)>0$, the following result holds true:

$$
\begin{aligned}
& \int_{0}^{\infty} e^{-s t}\left\{\int_{0}^{t} \tau^{d-1}(t-\tau)^{d^{\prime}-1}{ }_{2} F_{2}\left[\frac{1}{2}(a+b+1), d^{\mid \frac{1}{2} \tau s}\right] \times{ }_{2} F_{2}\left[\begin{array}{c}
a, b \\
\frac{1}{2}\left(a^{\prime}+b^{\prime}+1\right), d^{\prime}
\end{array} \mid \frac{1}{2}(t-\tau) s\right] \mathrm{d} \tau\right\} \mathrm{d} t \\
& =\frac{\Gamma(d) \Gamma\left(d^{\prime}\right)}{s^{d+d^{\prime}}} \Delta_{2}(a, b) \Delta_{2}\left(a^{\prime}, b^{\prime}\right) .
\end{aligned}
$$

Theorem 11. For $\mathfrak{R}(s)>0, \mathfrak{R}(d)>0$, and $\mathfrak{R}\left(d^{\prime}\right)>0$, the following result holds true:

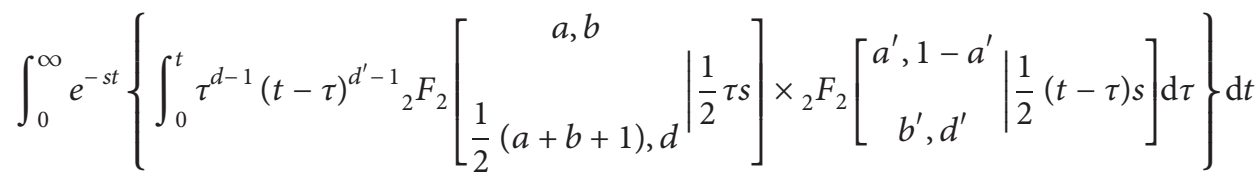

$$
\begin{aligned}
& =\frac{\Gamma(d) \Gamma\left(d^{\prime}\right)}{s^{d+d^{\prime}}} \Delta_{2}(a, b) \Delta_{3}\left(a^{\prime}, b^{\prime}\right) .
\end{aligned}
$$

Theorem 12. For $\mathfrak{R}(s)>0, \mathfrak{R}(d)>0$, and $\mathfrak{R}\left(d^{\prime}\right)>0$, the following result holds true: 


$$
\begin{aligned}
& \int_{0}^{\infty} e^{-s t}\left\{\int_{0}^{t} \tau^{d-1}(t-\tau)^{d^{\prime}-1}{ }_{2} F_{2}\left[\frac{1}{2}(a+b+1), d^{\mid \frac{1}{2} \tau s}\right] \times{ }_{2} F_{2}\left[\begin{array}{c}
a, b \\
a^{\prime}, b^{\prime} \\
1+a^{\prime}-b^{\prime}, d^{\prime}
\end{array} \mid-(t-\tau) s\right] \mathrm{d} \tau\right\} \mathrm{d} t \\
& =\frac{\Gamma(d) \Gamma\left(d^{\prime}\right)}{s^{d+d^{\prime}}} \Delta_{2}(a, b) \Delta_{4}\left(a^{\prime}, b^{\prime}\right) .
\end{aligned}
$$

Theorem 13. For $\mathfrak{R}(s)>0, \quad \mathfrak{R}(d)>0, \quad \mathfrak{R}\left(d^{\prime}\right)>0$, and $\mathfrak{R}\left(2 c^{\prime}-a^{\prime}-b^{\prime}\right)>-1$, the following result holds true:

$$
\begin{aligned}
& \int_{0}^{\infty} e^{-s t}\left\{\int_{0}^{t} \tau^{d-1}(t-\tau)^{d^{\prime}-1}{ }_{2} F_{2}\left[\frac{1}{2}(a+b+1), d^{\mid \frac{1}{2} \tau s}\right] \times{ }_{3} F_{3}\left[\begin{array}{c}
a, b \\
\frac{1}{2}\left(a^{\prime}+b^{\prime}+1\right), 2 c^{\prime}, d^{\prime}
\end{array} \mid(t-\tau) s\right] \mathrm{d} \tau\right\} \mathrm{d} t \\
& =\frac{\Gamma(d) \Gamma\left(d^{\prime}\right)}{s^{d+d^{\prime}}} \Delta_{2}(a, b) \Delta_{5}\left(a^{\prime}, b^{\prime}, c^{\prime}\right) .
\end{aligned}
$$

Theorem 14. For $\mathfrak{R}(s)>0, \quad \mathfrak{R}(d)>0, \quad \mathfrak{R}\left(d^{\prime}\right)>0$, and $\mathfrak{R}\left(a^{\prime}-2 b^{\prime}-2 c^{\prime}\right)>-2$, the following result holds true:

$$
\begin{aligned}
& \left.\int_{0}^{\infty} e^{-s t}\left\{\int_{0}^{t} \tau^{d-1}(t-\tau)^{d^{\prime}-1}{ }_{2} F_{2}\left[\frac{1}{2}(a+b+1), d^{\mid \frac{1}{2} \tau s}\right] \times{ }_{3} F_{3}\left[\begin{array}{c}
a, b \\
a^{\prime}, b^{\prime}, c^{\prime}
\end{array}\right](t-\tau) s\right] \mathrm{d} \tau\right\} \mathrm{d} t \\
& =\frac{\Gamma(d) \Gamma\left(d^{\prime}\right)}{s^{d+d^{\prime}}} \Delta_{2}(a, b) \Delta_{6}\left(a^{\prime}, b^{\prime}, c^{\prime}\right) .
\end{aligned}
$$

Theorem 15. For $\mathfrak{R}(s)>0, \mathfrak{R}(d)>0$, and $\mathfrak{R}\left(d^{\prime}\right)>0$, the following result holds true:

$$
\begin{aligned}
& \int_{0}^{\infty} e^{-s t}\left\{\int_{0}^{t} \tau^{d-1}(t-\tau)^{d^{\prime}-1}{ }_{2} F_{2}\left[\begin{array}{c}
a, b \\
\frac{1}{2}(a+b+1), d
\end{array} \mid \frac{1}{2} \tau s\right] \times{ }_{3} F_{3}\left[\begin{array}{c}
a^{\prime}, 1-a^{\prime}, c^{\prime} \\
e^{\prime}, 1+2 c^{\prime}-e^{\prime}, d^{\prime}
\end{array} \mid(t-\tau) s\right] \mathrm{d} \tau\right\} \mathrm{d} t \\
& =\frac{\Gamma(d) \Gamma\left(d^{\prime}\right)}{s^{d+d^{\prime}}} \Delta_{2}(a, b) \Delta_{7}\left(a^{\prime}, c^{\prime}, e^{\prime}\right) .
\end{aligned}
$$

Theorem 16. For $\mathfrak{R}(s)>0, \mathfrak{R}(d)>0$, and $\mathfrak{R}\left(d^{\prime}\right)>0$, the following result holds true: 


$$
\begin{aligned}
& \left.\int_{0}^{\infty} e^{-s t}\left\{\int_{0}^{t} \tau^{d-1}(t-\tau)^{d^{\prime}-1}{ }_{2} F_{2}\left[\frac{1}{2}(a+b+1), d^{\mid \frac{1}{2} \tau s}\right] \times{ }_{4} F_{4}\left[\begin{array}{c}
a, b \\
a^{\prime}, 1+\frac{1}{2} a^{\prime}, b^{\prime}, c^{\prime} \\
\frac{1}{2} a^{\prime}, a^{\prime}-b^{\prime}+1, a^{\prime}-c^{\prime}+1, d^{\prime}
\end{array}\right]-(t-\tau) s\right] \mathrm{d} \tau\right\} \mathrm{d} t \\
& =\frac{\Gamma(d) \Gamma\left(d^{\prime}\right)}{s^{d+d^{\prime}} \Delta_{2}(a, b) \Delta_{8}\left(a^{\prime}, b^{\prime}, c^{\prime}\right) .}
\end{aligned}
$$

Theorem 17. For $\mathfrak{R}(s)>0, \mathfrak{R}(d)>0, \quad \mathfrak{R}\left(f^{\prime}\right)>0$, and

$\mathfrak{R}\left(a^{\prime}-c^{\prime}-d^{\prime}-e^{\prime}+1\right)>0$, the following result holds true:

$$
\begin{aligned}
& \int_{0}^{\infty} e^{-s t}\left\{\int_{0}^{t} \tau^{d-1}(t-\tau)^{f^{\prime}-1}{ }_{2} F_{2}\left[\frac{1}{2}(a+b+1), d^{\mid \frac{1}{2} \tau s}\right] \times{ }_{5} F_{5}\left[\begin{array}{c}
a^{\prime}, 1+\frac{1}{2} a^{\prime}, c^{\prime}, d^{\prime}, e^{\prime} \\
\frac{1}{2} a^{\prime}, a^{\prime}-c^{\prime}+1, a^{\prime}-d^{\prime}+1, a^{\prime}-e^{\prime}+1, f^{\prime}
\end{array} \mid(t-\tau) s\right] \mathrm{d} \tau\right\} \mathrm{d} t \\
& =\frac{\Gamma(d) \Gamma\left(f^{\prime}\right)}{s^{d+f^{\prime}}} \Delta_{2}(a, b) \Delta_{9}\left(a^{\prime}, c^{\prime}, d^{\prime}, e^{\prime}\right) .
\end{aligned}
$$

Theorem 18. For $\mathfrak{R}(s)>0, \mathfrak{R}(d)>0$, and $\mathfrak{R}\left(d^{\prime}\right)>0$, the following result holds true:

$$
\begin{aligned}
& \int_{0}^{\infty} e^{-s t}\left\{\int_{0}^{t} \tau^{d-1}(t-\tau)^{d^{\prime}-1}{ }_{2} F_{2}\left[\begin{array}{c}
a, 1-a \\
b, d
\end{array} \mid \frac{1}{2} \tau s\right] \times{ }_{2} F_{2}\left[\begin{array}{c}
a^{\prime}, 1-a^{\prime} \\
b^{\prime}, d^{\prime}
\end{array} \mid \frac{1}{2}(t-\tau) s\right] \mathrm{d} \tau\right\} \mathrm{d} t \\
& =\frac{\Gamma(d) \Gamma\left(d^{\prime}\right)}{s^{d+d^{\prime}}} \Delta_{3}(a, b) \Delta_{3}\left(a^{\prime}, b^{\prime}\right) .
\end{aligned}
$$

Theorem 19. For $\mathfrak{R}(s)>0, \mathfrak{R}(d)>0$, and $\mathfrak{R}\left(d^{\prime}\right)>0$, the following result holds true:

$$
\begin{aligned}
& \int_{0}^{\infty} e^{-s t}\left\{\int_{0}^{t} \tau^{d-1}(t-\tau)^{d^{\prime}-1}{ }_{2} F_{2}\left[\begin{array}{c}
a, 1-a \\
b, d
\end{array} \mid \frac{1}{2} \tau s\right] \times{ }_{2} F_{2}\left[\begin{array}{c}
a^{\prime}, b^{\prime} \\
1+a^{\prime}-b^{\prime}, d^{\prime}
\end{array} \mid-(t-\tau) s\right] \mathrm{d} \tau\right\} \mathrm{d} t \\
& =\frac{\Gamma(d) \Gamma\left(d^{\prime}\right)}{s^{d+d^{\prime}}} \Delta_{3}(a, b) \Delta_{4}\left(a^{\prime}, b^{\prime}\right) .
\end{aligned}
$$

Theorem 20. For $\mathfrak{R}(s)>0, \quad \mathfrak{R}(d)>0, \quad \mathfrak{R}\left(d^{\prime}\right)>0$, and $\mathfrak{R}\left(2 c^{\prime}-a^{\prime}-b^{\prime}\right)>-1$, the following result holds true: 


$$
\begin{aligned}
& \int_{0}^{\infty} e^{-s t}\left\{\int_{0}^{t} \tau^{d-1}(t-\tau)^{d^{\prime}-1}{ }_{2} F_{2}\left[\begin{array}{c}
a, 1-a \\
b, d
\end{array} \mid \frac{1}{2} \tau s\right] \times{ }_{3} F_{3}\left[\begin{array}{c}
a^{\prime}, b^{\prime}, c^{\prime} \\
\frac{1}{2}\left(a^{\prime}+b^{\prime}+1\right), 2 c^{\prime}, d^{\prime}
\end{array} \mid(t-\tau) s\right] \mathrm{d} \tau\right\} \mathrm{d} t \\
& =\frac{\Gamma(d) \Gamma\left(d^{\prime}\right)}{s^{d+d^{\prime}}} \Delta_{3}(a, b) \Delta_{5}\left(a^{\prime}, b^{\prime}, c^{\prime}\right) .
\end{aligned}
$$

Theorem 21. For $\mathfrak{R}(s)>0, \quad \mathfrak{R}(d)>0, \quad \mathfrak{R}\left(d^{\prime}\right)>0$, and $\mathfrak{R}\left(a^{\prime}-2 b^{\prime}-c^{\prime}\right)>-2$, the following result holds true:

$$
\begin{aligned}
& \int_{0}^{\infty} e^{-s t}\left\{\int_{0}^{t} \tau^{d-1}(t-\tau)^{d^{\prime}-1}{ }_{2} F_{2}\left[\begin{array}{c}
a, 1-a \\
b, d
\end{array} \mid \frac{1}{2} \tau s\right] \times{ }_{3} F_{3}\left[\begin{array}{c}
a^{\prime}, b^{\prime}, c^{\prime} \\
1+a^{\prime}-b^{\prime}, 1+a^{\prime}-c^{\prime}, d^{\prime}
\end{array} \mid(t-\tau) s\right] \mathrm{d} \tau\right\} \mathrm{d} t \\
& =\frac{\Gamma(d) \Gamma\left(d^{\prime}\right)}{s^{d+d^{\prime}}} \Delta_{3}(a, b) \Delta_{6}\left(a^{\prime}, b^{\prime}, c^{\prime}\right) .
\end{aligned}
$$

Theorem 22. For $\mathfrak{R}(s)>0, \mathfrak{R}(d)>0$, and $\mathfrak{R}\left(d^{\prime}\right)>0$, the following result holds true:

$$
\begin{aligned}
& \int_{0}^{\infty} e^{-s t}\left\{\int_{0}^{t} \tau^{d-1}(t-\tau)^{d^{\prime}}{ }_{2} F_{2}\left[\begin{array}{c}
a, 1-a \\
b, d
\end{array} \mid \frac{1}{2} \tau s\right] \times{ }_{3} F_{3}\left[\begin{array}{c}
a^{\prime}, 1-a^{\prime}, c^{\prime} \\
e^{\prime}, 1+2 c^{\prime}-e^{\prime}, d^{\prime}
\end{array} \mid(t-\tau) s\right] \mathrm{d} \tau\right\} \mathrm{d} t \\
& =\frac{\Gamma(d) \Gamma\left(d^{\prime}\right)}{s^{d+d^{\prime}}} \Delta_{3}(a, b) \Delta_{7}\left(a^{\prime}, c^{\prime}, e^{\prime}\right) .
\end{aligned}
$$

Theorem 23. For $\mathfrak{R}(s)>0, \mathfrak{R}(d)>0$, and $\mathfrak{R}\left(d^{\prime}\right)>0$, the following result holds true:

$$
\begin{aligned}
& \left.\int_{0}^{\infty} e^{-s t}\left\{\int_{0}^{t} \tau^{d-1}(t-\tau)^{d^{\prime}-1}{ }_{2} F_{2}\left[\begin{array}{c}
a, 1-a \\
b, d
\end{array} \mid \frac{1}{2} \tau s\right] \times{ }_{4} F_{4}\left[\begin{array}{c}
a^{\prime}, 1+\frac{1}{2} a^{\prime}, b^{\prime}, c^{\prime} \\
\frac{1}{2} a^{\prime}, a^{\prime}-b^{\prime}+1, a^{\prime}-c^{\prime}+1, d^{\prime}
\end{array}\right]-(t-\tau) s\right] \mathrm{d} \tau\right\} \mathrm{d} t \\
& =\frac{\Gamma(d) \Gamma\left(d^{\prime}\right)}{s^{d+d^{\prime}}} \Delta_{3}(a, b) \Delta_{8}\left(a^{\prime}, b^{\prime}, c^{\prime}\right) .
\end{aligned}
$$

Theorem 24. For $\mathfrak{R}(s)>0, \quad \mathfrak{R}(d)>0, \quad \mathfrak{R}\left(f^{\prime}\right)>0$, and $\mathfrak{R}\left(a^{\prime}-c^{\prime}-d^{\prime}-e^{\prime}+1\right)>0$, the following result holds true: 


$$
\begin{aligned}
& \left.\int_{0}^{\infty} e^{-s t}\left\{\int_{0}^{t} \tau^{d-1}(t-\tau)^{f^{\prime}-1}{ }_{2} F_{2}\left[\begin{array}{c}
a, 1-a \\
b, d
\end{array} \mid \frac{1}{2} \tau s\right] \times{ }_{5} F_{5}\left[\begin{array}{c}
a^{\prime}, 1+\frac{1}{2} a^{\prime}, c^{\prime}, d^{\prime}, e^{\prime} \\
\frac{1}{2} a^{\prime}, a^{\prime}-c^{\prime}+1, a^{\prime}-d^{\prime}+1, a^{\prime}-e^{\prime}+1, f^{\prime}
\end{array}\right](t-\tau) s\right] \mathrm{d} \tau\right\} \mathrm{d} t \\
& =\frac{\Gamma(d) \Gamma\left(f^{\prime}\right)}{s^{d+f^{\prime}} \Delta_{3}(a, b) \Delta_{9}\left(a^{\prime}, c^{\prime}, d^{\prime}, e^{\prime}\right) .}
\end{aligned}
$$

Theorem 25. For $\mathfrak{R}(s)>0, \mathfrak{R}(d)>0$, and $\mathfrak{R}\left(d^{\prime}\right)>0$, the following result holds true:

$$
\begin{aligned}
& \int_{0}^{\infty} e^{-s t}\left\{\int_{0}^{t} \tau^{d-1}(t-\tau)^{d^{\prime}-1}{ }_{2} F_{2}\left[\begin{array}{c}
a, b \\
1+a-b, d
\end{array} \mid-\tau s\right] \times{ }_{2} F_{2}\left[\begin{array}{c}
a^{\prime}, b^{\prime} \\
1+a^{\prime}-b^{\prime}, d^{\prime}
\end{array} \mid-(t-\tau) s\right] \mathrm{d} \tau\right\} \mathrm{d} t \\
& =\frac{\Gamma(d) \Gamma\left(d^{\prime}\right)}{s^{d+d^{\prime}}} \Delta_{4}(a, b) \Delta_{4}\left(a^{\prime}, b^{\prime}\right) .
\end{aligned}
$$

Theorem 26. For $\mathfrak{R}(s)>0, \quad \mathfrak{R}(d)>0, \quad \mathfrak{R}\left(d^{\prime}\right)>0$, and $\mathfrak{R}\left(2 c^{\prime}-a^{\prime}-b^{\prime}\right)>-2$, the following result holds true:

$$
\begin{aligned}
& \left.\left.\int_{0}^{\infty} e^{-s t}\left\{\int_{0}^{t} \tau^{d-1}(t-\tau)^{d^{\prime}-1}{ }_{2} F_{2}\left[\begin{array}{c}
a, b \\
1+a-b, d
\end{array}\right] \mid-\tau s\right] \times{ }_{3} F_{3}\left[\begin{array}{c}
a^{\prime}, b^{\prime}, c^{\prime} \\
\frac{1}{2}\left(a^{\prime}+b^{\prime}+1\right), 2 c^{\prime}, d^{\prime}
\end{array}\right] \mathrm{I}(t-\tau) s\right] \mathrm{~d} \tau\right\} \mathrm{d} t \\
& =\frac{\Gamma(d) \Gamma\left(d^{\prime}\right)}{s^{d+d^{\prime}}} \Delta_{4}(a, b) \Delta_{5}\left(a^{\prime}, b^{\prime}, c^{\prime}\right) .
\end{aligned}
$$

Theorem 27. For $\mathfrak{R}(s)>0, \quad \mathfrak{R}(d)>0, \quad \mathfrak{R}\left(d^{\prime}\right)>0$, and $\mathfrak{R}\left(a^{\prime}-2 b^{\prime}-c^{\prime}\right)>-2$, the following result holds true:

$$
\begin{aligned}
& \int_{0}^{\infty} e^{-s t}\left\{\int_{0}^{t} \tau^{d-1}(t-\tau)^{d^{\prime}-1}{ }_{2} F_{2}\left[\begin{array}{c}
a, b \\
1+a-b, d
\end{array} \mid-\tau s\right] \times{ }_{3} F_{3}\left[\begin{array}{c}
a^{\prime}, b^{\prime}, c^{\prime} \\
1+a^{\prime}-b^{\prime}, 1+a^{\prime}-c^{\prime}, d^{\prime}
\end{array} \mid(t-\tau) s\right] \mathrm{d} \tau\right\} \mathrm{d} t \\
& =\frac{\Gamma(d) \Gamma\left(d^{\prime}\right)}{s^{d+d^{\prime}}} \Delta_{4}(a, b) \Delta_{6}\left(a^{\prime}, b^{\prime}, c^{\prime}\right) .
\end{aligned}
$$

Theorem 28. For $\mathfrak{R}(s)>0, \mathfrak{R}(d)>0$, and $\mathfrak{R}\left(d^{\prime}\right)>0$, the following result holds true:

$$
\begin{aligned}
& \int_{0}^{\infty} e^{-s t}\left\{\int_{0}^{t} \tau^{d-1}(t-\tau)^{d^{\prime}-1}{ }_{2} F_{2}\left[\begin{array}{c}
a, b \\
1+a-b, d
\end{array} \mid-\tau s\right] \times{ }_{3} F_{3}\left[\begin{array}{c}
a^{\prime}, 1-a^{\prime}, c^{\prime} \\
e^{\prime}, 1+2 c^{\prime}-e^{\prime}, d^{\prime}
\end{array} \mid(t-\tau) s\right] \mathrm{d} \tau\right\} \mathrm{d} t \\
& =\frac{\Gamma(d) \Gamma\left(d^{\prime}\right)}{s^{d+d^{\prime}}} \Delta_{4}(a, b) \Delta_{7}\left(a^{\prime}, c^{\prime}, e^{\prime}\right) .
\end{aligned}
$$


Theorem 29. For $\mathfrak{R}(s)>0, \mathfrak{R}(d)>0$, and $\mathfrak{R}\left(d^{\prime}\right)>0$, the following result holds true:

$$
\begin{aligned}
& \left.\int_{0}^{\infty} e^{-s t}\left\{\int_{0}^{t} \tau^{d-1}(t-\tau)^{d^{\prime}-1}{ }_{2} F_{2}\left[\begin{array}{c}
a, b \\
1+a-b, d
\end{array} \mid-\tau s\right] \times{ }_{4} F_{4}\left[\begin{array}{c}
a^{\prime}, 1+\frac{1}{2} a^{\prime}, b^{\prime}, c^{\prime} \\
\frac{1}{2} a^{\prime}, a^{\prime}-b^{\prime}+1, a^{\prime}-c^{\prime}+1, d^{\prime}
\end{array}\right]-(t-\tau) s\right] \mathrm{d} \tau\right\} \mathrm{d} t \\
& =\frac{\Gamma(d) \Gamma\left(d^{\prime}\right)}{s^{d+d^{\prime}} \Delta_{4}(a, b) \Delta_{8}\left(a^{\prime}, b^{\prime}, c^{\prime}\right) .}
\end{aligned}
$$

Theorem 30. For $\mathfrak{R}(s)>0, \quad \mathfrak{R}(d)>0, \quad \mathfrak{R}\left(f^{\prime}\right)>0$, and $\mathfrak{R}\left(a^{\prime}-c^{\prime}-d^{\prime}-e^{\prime}+1\right)>0$, the following result holds true:

$$
\begin{aligned}
& \left.\int_{0}^{\infty} e^{-s t}\left\{\int_{0}^{t} \tau^{d-1}(t-\tau){ }^{f^{\prime}-1}{ }_{2} F_{2}\left[\begin{array}{c}
a, b \\
1+a-b, d
\end{array} \mid-\tau s\right] \times{ }_{5} F_{5}\left[\begin{array}{c}
a^{\prime}, 1+\frac{1}{2} a^{\prime}, c^{\prime}, d^{\prime}, e^{\prime} \\
\frac{1}{2} a^{\prime}, a^{\prime}-c^{\prime}+1, a^{\prime}-d^{\prime}+1, a^{\prime}-e^{\prime}+1, f^{\prime}
\end{array}\right](t-\tau) s\right] \mathrm{d} \tau\right\} \mathrm{d} t \\
& =\frac{\Gamma(d) \Gamma\left(f^{\prime}\right)}{s^{d+f^{\prime}} \Delta_{4}(a, b) \Delta_{9}\left(a^{\prime}, c^{\prime}, d^{\prime}, e^{\prime}\right) .}
\end{aligned}
$$

Theorem 31. For $\mathfrak{R}(s)>0, \quad \mathfrak{R}(d)>0, \quad \mathfrak{R}\left(d^{\prime}\right)>0$, $\mathfrak{R}(2 c-a-b)>-1$, and $\mathfrak{R}\left(2 c^{\prime}-a^{\prime}-b^{\prime}\right)>-1$, the following result holds true:

$$
\begin{aligned}
& \left.\int_{0}^{\infty} e^{-s t}\left\{\int_{0}^{t} \tau^{d-1}(t-\tau)^{d^{\prime}-1}{ }_{3} F_{3}\left[\begin{array}{c}
a, b, c \\
\frac{1}{2}(a+b+1), 2 c, d
\end{array}\right] \tau s\right] \times{ }_{3} F_{3}\left[\begin{array}{c}
a^{\prime}, b^{\prime}, c^{\prime} \\
\frac{1}{2}\left(a^{\prime}+b^{\prime}+1\right), 2 c^{\prime}, d^{\prime}
\end{array} \mid(t-\tau) s\right] \mathrm{d} \tau\right\} \mathrm{d} t \\
& =\frac{\Gamma(d) \Gamma\left(d^{\prime}\right)}{s^{d+d^{\prime}}} \Delta_{5}(a, b, c) \Delta_{5}\left(a^{\prime}, b^{\prime}, c^{\prime}\right) \text {. }
\end{aligned}
$$

Theorem 32. For $\mathfrak{R}(s)>0, \quad \mathfrak{R}(d)>0, \quad \mathfrak{R}\left(d^{\prime}\right)>0$, $\mathfrak{R}(2 c-a-b)>-1$, and $\mathfrak{R}\left(a^{\prime}-2 b^{\prime}-c^{\prime}\right)>-2$, the following result holds true:

$$
\begin{aligned}
& \int_{0}^{\infty} e^{-s t}\left\{\int_{0}^{t} \tau^{d-1}(t-\tau)^{d^{\prime}-1}{ }_{3} F_{3}\left[\begin{array}{c}
a, b, c \\
\frac{1}{2}(a+b+1), 2 c, d
\end{array} \mid \tau s\right] \times{ }_{3} F_{3}\left[\begin{array}{c}
a^{\prime}, b^{\prime}, c^{\prime} \\
1+a^{\prime}-b^{\prime}, 1+a^{\prime}-c^{\prime}, d^{\prime}
\end{array} \mid(t-\tau) s\right] \mathrm{d} \tau\right\} \mathrm{d} t \\
& =\frac{\Gamma(d) \Gamma\left(d^{\prime}\right)}{s^{d+d^{\prime}}} \Delta_{5}(a, b, c) \Delta_{6}\left(a^{\prime}, b^{\prime}, c^{\prime}\right) .
\end{aligned}
$$


Theorem 33. For $\mathfrak{R}(s)>0, \quad \mathfrak{R}(d)>0, \quad \mathfrak{R}\left(d^{\prime}\right)>0$, and $\mathfrak{R}\left(2 c^{\prime}-a^{\prime}-b^{\prime}\right)>-1$, the following result holds true:

$$
\begin{aligned}
& \int_{0}^{\infty} e^{-s t}\left\{\int_{0}^{t} \tau^{d-1}(t-\tau)^{d^{\prime}-1}{ }_{3} F_{3}\left[\begin{array}{c}
a, b, c \\
\frac{1}{2}(a+b+1), 2 c, d
\end{array} \mid \tau s\right] \times{ }_{3} F_{3}\left[\begin{array}{c}
a^{\prime}, 1-a^{\prime}, c^{\prime} \\
e^{\prime}, 1+2 c^{\prime}-e^{\prime}, d^{\prime}
\end{array} \mid(t-\tau) s\right] \mathrm{d} \tau\right\} \mathrm{d} t \\
& =\frac{\Gamma(d) \Gamma\left(d^{\prime}\right)}{s^{d+d^{\prime}}} \Delta_{5}(a, b, c) \Delta_{7}\left(a^{\prime}, c^{\prime}, e^{\prime}\right) .
\end{aligned}
$$

Theorem 34. For $\mathfrak{R}(s)>0, \quad \mathfrak{R}(d)>0, \quad \mathfrak{R}\left(d^{\prime}\right)>0$, and $\mathfrak{R}(2 c-a-b)>-1$, the following result holds true:

$$
\begin{aligned}
& \left.\int_{0}^{\infty} e^{-s t}\left\{\int_{0}^{t} \tau^{d-1}(t-\tau)^{d^{\prime}-1}{ }_{3} F_{3}\left[\begin{array}{c}
a, b, c \\
\frac{1}{2}(a+b+1), 2 c, d
\end{array} \mid \tau s\right] \times{ }_{4} F_{4}\left[\begin{array}{c}
a^{\prime}, 1+\frac{1}{2} a^{\prime}, b^{\prime}, c^{\prime} \\
\frac{1}{2} a^{\prime}, a^{\prime}-b^{\prime}+1, a^{\prime}-c^{\prime}+1, d^{\prime}
\end{array}\right]-(t-\tau) s\right] \mathrm{d} \tau\right\} \mathrm{d} t \\
& =\frac{\Gamma(d) \Gamma\left(d^{\prime}\right)}{s^{d+d^{\prime}}} \Delta_{5}(a, b, c) \Delta_{8}\left(a^{\prime}, b^{\prime}, c^{\prime}\right) .
\end{aligned}
$$

Theorem 35. For $\mathfrak{R}(s)>0, \quad \mathfrak{R}(d)>0, \quad \mathfrak{R}\left(f^{\prime}\right)>0$, $\Re(2 c-a-b)>-1$, and $\mathfrak{R}\left(a^{\prime}-c^{\prime}-d^{\prime}-e^{\prime}+1\right)>0$, the following result holds true:

$$
\begin{aligned}
& \left.\int_{0}^{\infty} e^{-s t}\left\{\int_{0}^{t} \tau^{d-1}(t-\tau)^{f^{\prime}-1}{ }_{3} F_{3}\left[\frac{1}{2}(a+b+1), 2 c, d^{\mid \tau s}\right] \times{ }_{5} F_{5}\left[\begin{array}{c}
a, b \\
a^{\prime}, 1+\frac{1}{2} a^{\prime}, c^{\prime}, d^{\prime}, e^{\prime} \\
\frac{1}{2} a^{\prime}, a^{\prime}-c^{\prime}+1, a^{\prime}-d^{\prime}+1, a^{\prime}-e^{\prime}+1, f^{\prime}
\end{array}\right](t-\tau) s\right] \mathrm{d} \tau\right\} \mathrm{d} t \\
& =\frac{\Gamma(d) \Gamma\left(f^{\prime}\right)}{s^{d+f^{\prime}}} \Delta_{5}(a, b, c) \Delta_{9}\left(a^{\prime}, c^{\prime}, d^{\prime}, e^{\prime}\right) .
\end{aligned}
$$

Theorem 36. For $\mathfrak{R}(s)>0, \quad \mathfrak{R}(d)>0, \quad \mathfrak{R}\left(d^{\prime}\right)>0$, $\mathfrak{R}(a-2 b-2 c)>-2$, and $\mathfrak{R}\left(a^{\prime}-2 b^{\prime}-c^{\prime}\right)>-2$, the following result holds true:

$$
\begin{aligned}
& \int_{0}^{\infty} e^{-s t}\left\{\int_{0}^{t} \tau^{d-1}(t-\tau)^{d^{\prime}-1}{ }_{3} F_{3}\left[\begin{array}{c}
a, b, c \\
1+a-b, 1+a-c, d
\end{array} \mid \tau s\right] \times{ }_{3} F_{3}\left[\begin{array}{c}
a^{\prime}, b^{\prime}, c^{\prime} \\
1+a^{\prime}-b^{\prime}, 1+a^{\prime}-c^{\prime}, d^{\prime}
\end{array} \mid(t-\tau) s\right] \mathrm{d} \tau\right\} \mathrm{d} t \\
& =\frac{\Gamma(d) \Gamma\left(d^{\prime}\right)}{s^{d+d^{\prime}}} \Delta_{6}(a, b, c) \Delta_{6}\left(a^{\prime}, b^{\prime}, c^{\prime}\right) .
\end{aligned}
$$


Theorem 37. For $\mathfrak{R}(s)>0, \mathfrak{R}(d)>0, \quad \mathfrak{R}\left(d^{\prime}\right)>0$, and

$\mathfrak{R}(a-2 b-2 c)>-2$, the following result holds true:

$$
\begin{aligned}
& \int_{0}^{\infty} e^{-s t}\left\{\int_{0}^{t} \tau^{d-1}(t-\tau)^{d^{\prime}-1}{ }_{3} F_{3}\left[\begin{array}{c}
a, b, c \\
1+a-b, 1+a-c, d
\end{array} \mid \tau s\right] \times{ }_{3} F_{3}\left[\begin{array}{c}
a^{\prime}, 1-a^{\prime}, c^{\prime} \\
e^{\prime}, 1+2 c^{\prime}-e^{\prime}, d^{\prime}
\end{array} \mid(t-\tau) s\right] \mathrm{d} \tau\right\} \mathrm{d} t \\
& =\frac{\Gamma(d) \Gamma\left(d^{\prime}\right)}{s^{d+d^{\prime}}} \Delta_{6}(a, b, c) \Delta_{7}\left(a^{\prime}, c^{\prime}, e^{\prime}\right) .
\end{aligned}
$$

Theorem 38. For $\mathfrak{R}(s)>0, \quad \mathfrak{R}(d)>0, \quad \mathfrak{R}\left(d^{\prime}\right)>0$, and $\mathfrak{R}(a-2 b-2 c)>-2$, the following result holds true:

$$
\begin{aligned}
& \left.\left.\int_{0}^{\infty} e^{-s t}\left\{\int_{0}^{t} \tau^{d-1}(t-\tau)^{d^{\prime}-1}{ }_{3} F_{3}\left[\begin{array}{c}
a, b, c \\
1+a-b, 1+a-c, d
\end{array}\right] \tau s\right] \times{ }_{4} F_{4}\left[\begin{array}{c}
a^{\prime}, 1+\frac{1}{2} a^{\prime}, b^{\prime}, c^{\prime} \\
\frac{1}{2} a^{\prime}, a^{\prime}-b^{\prime}+1, a^{\prime}-c^{\prime}+1, d^{\prime}
\end{array}\right]-(t-\tau) s\right] \mathrm{~d} \tau\right\} \mathrm{d} t \\
& =\frac{\Gamma(d) \Gamma\left(d^{\prime}\right)}{s^{d+d^{\prime}} \Delta_{6}(a, b, c) \Delta_{8}\left(a^{\prime}, b^{\prime}, c^{\prime}\right) .}
\end{aligned}
$$

Theorem 39. For $\mathfrak{R}(s)>0, \quad \mathfrak{R}(d)>0, \quad \mathfrak{R}\left(f^{\prime}\right)>0$, $\Re(a-2 b-2 c)>-2$, and $\mathfrak{R}\left(a^{\prime}-c^{\prime}-d^{\prime}-e^{\prime}+1\right)>0$, the following result holds true:

$$
\begin{aligned}
& \left.\left.\int_{0}^{\infty} e^{-s t}\left\{\int_{0}^{t} \tau^{d-1}(t-\tau) f^{f^{\prime}-1}{ }_{3} F_{3}\left[\begin{array}{c}
a, b, c \\
1+a-b, 1+a-c, d
\end{array}\right] \tau s\right] \times{ }_{5} F_{5}\left[\begin{array}{c}
a^{\prime}, 1+\frac{1}{2} a^{\prime}, c^{\prime}, d^{\prime}, e^{\prime} \\
\frac{1}{2} a^{\prime}, a^{\prime}-c^{\prime}+1, a^{\prime}-d^{\prime}+1, a^{\prime}-e^{\prime}+1, f^{\prime}
\end{array}\right](t-\tau) s\right] \mathrm{~d} \tau\right\} \mathrm{d} t \\
& =\frac{\Gamma(d) \Gamma\left(f^{\prime}\right)}{s^{d+f^{\prime}} \Delta_{6}(a, b, c) \Delta_{9}\left(a^{\prime}, c^{\prime}, d^{\prime}, e^{\prime}\right) .}
\end{aligned}
$$

Theorem 40. For $\mathfrak{R}(s)>0, \mathfrak{R}(d)>0$, and $\mathfrak{R}\left(d^{\prime}\right)>0$, the following result holds true:

$$
\begin{aligned}
& \int_{0}^{\infty} e^{-s t}\left\{\int_{0}^{t} \tau^{d-1}(t-\tau)^{d^{\prime}-1}{ }_{3} F_{3}\left[\begin{array}{c}
a, 1-a, c \\
e, 1+2 c-e, d
\end{array} \mid \tau s\right] \times{ }_{3} F_{3}\left[\begin{array}{c}
a^{\prime}, 1-a^{\prime}, c^{\prime} \\
e^{\prime}, 1+2 c^{\prime}-e^{\prime}, d^{\prime}
\end{array} \mid(t-\tau) s\right] \mathrm{d} \tau\right\} \mathrm{d} t \\
& =\frac{\Gamma(d) \Gamma\left(d^{\prime}\right)}{s^{d+d^{\prime}}} \Delta_{7}(a, c, e) \Delta_{7}\left(a^{\prime}, c^{\prime}, e^{\prime}\right) .
\end{aligned}
$$

Theorem 41. For $\mathfrak{R}(s)>0, \mathfrak{R}(d)>0$, and $\mathfrak{R}\left(d^{\prime}\right)>0$, the following result holds true: 


$$
\begin{aligned}
& \left.\left.\int_{0}^{\infty} e^{-s t}\left\{\int_{0}^{t} \tau^{d-1}(t-\tau)^{d^{\prime}-1}{ }_{3} F_{3}\left[\begin{array}{c}
a, 1-a, c \\
e, 1+2 c-e, d
\end{array}\right] \tau s\right] \times{ }_{4} F_{4}\left[\begin{array}{c}
a^{\prime}, 1+\frac{1}{2} a^{\prime}, b^{\prime}, c^{\prime} \\
\frac{1}{2} a^{\prime}, a^{\prime}-b^{\prime}+1, a^{\prime}-c^{\prime}+1, d^{\prime}
\end{array}\right]-(t-\tau) s\right] \mathrm{~d} \tau\right\} \mathrm{d} t \\
& =\frac{\Gamma(d) \Gamma\left(d^{\prime}\right)}{s^{d+d^{\prime}}} \Delta_{7}(a, c, e) \Delta_{8}\left(a^{\prime}, b^{\prime}, c^{\prime}\right) .
\end{aligned}
$$

Theorem 42. For $\mathfrak{R}(s)>0, \mathfrak{R}(d)>0, \quad \mathfrak{R}\left(f^{\prime}\right)>0$, and $\mathfrak{R}\left(a^{\prime}-c^{\prime}-d^{\prime}-e^{\prime}+1\right)>0$, the following result holds true:

$$
\begin{aligned}
& \left.\int_{0}^{\infty} e^{-s t}\left\{\int_{0}^{t} \tau^{d-1}(t-\tau)^{f^{\prime}-1}{ }_{3} F_{3}\left[\begin{array}{c}
a, 1-a, c \\
e, 1+2 c-e, d
\end{array} \mid \tau s\right] \times{ }_{5} F_{5}\left[\begin{array}{c}
a^{\prime}, 1+\frac{1}{2} a^{\prime}, c^{\prime}, d^{\prime}, e^{\prime} \\
\frac{1}{2} a^{\prime}, a^{\prime}-c^{\prime}+1, a^{\prime}-d^{\prime}+1, a^{\prime}-e^{\prime}+1, f^{\prime}
\end{array}\right](t-\tau) s\right] \mathrm{d} \tau\right\} \mathrm{d} t \\
& =\frac{\Gamma(d) \Gamma\left(f^{\prime}\right)}{s^{d+f^{\prime}}} \Delta_{7}(a, c, e) \Delta_{9}\left(a^{\prime}, c^{\prime}, d^{\prime}, e^{\prime}\right) .
\end{aligned}
$$

Theorem 43. For $\mathfrak{R}(s)>0, \mathfrak{R}(d)>0$, and $\mathfrak{R}\left(d^{\prime}\right)>0$, the following result holds true:

$$
\begin{aligned}
& \left.\left.\int_{0}^{\infty} e^{-s t}\left\{\int_{0}^{t} \tau^{d-1}(t-\tau)^{d^{\prime}-1}{ }_{4} F_{4}\left[\begin{array}{c}
a, 1+\frac{1}{2} a, b, c \\
\frac{1}{2} a, a-b+1, a-c+1, d
\end{array}\right]-\tau s\right] \times{ }_{4} F_{4}\left[\begin{array}{c}
a^{\prime}, 1+\frac{1}{2} a^{\prime}, b^{\prime}, c^{\prime} \\
\frac{1}{2} a^{\prime}, a^{\prime}-b^{\prime}+1, a^{\prime}-c^{\prime}+1, d^{\prime}
\end{array}\right]-(t-\tau) s\right] \mathrm{~d} \tau\right\} \mathrm{d} t \\
& =\frac{\Gamma(d) \Gamma\left(d^{\prime}\right)}{s^{d+d^{\prime}}} \Delta_{8}(a, b, c) \Delta_{8}\left(a^{\prime}, b^{\prime}, c^{\prime}\right) .
\end{aligned}
$$

Theorem 44. For $\mathfrak{R}(s)>0, \mathfrak{R}(d)>0, \quad \mathfrak{R}\left(f^{\prime}\right)>0$, and $\mathfrak{R}\left(a^{\prime}-c^{\prime}-d^{\prime}-e^{\prime}+1\right)>0$, the following result holds true:

$$
\begin{aligned}
& \left.\left.\int_{0}^{\infty} e^{-s t}\left\{\int_{0}^{t} \tau^{d-1}(t-\tau)^{f^{\prime}-1}{ }_{4} F_{4}\left[\begin{array}{c}
a, 1+\frac{1}{2} a, b, c \\
\frac{1}{2} a, a-b+1, a-c+1, d
\end{array}\right]-\tau s\right] \times{ }_{5} F_{5}\left[\begin{array}{c}
a^{\prime}, 1+\frac{1}{2} a^{\prime}, c^{\prime}, d^{\prime}, e^{\prime} \\
\frac{1}{2} a^{\prime}, a^{\prime}-c^{\prime}+1, a^{\prime}-d^{\prime}+1, a^{\prime}-e^{\prime}+1, f^{\prime}
\end{array}\right](t-\tau) s\right] \mathrm{~d} \tau\right\} \mathrm{d} t \\
& =\frac{\Gamma(d) \Gamma\left(f^{\prime}\right)}{s^{d+f^{\prime}}} \Delta_{8}(a, c, e) \Delta_{9}\left(a^{\prime}, c^{\prime}, d^{\prime}, e^{\prime}\right) .
\end{aligned}
$$


Theorem 45. For $\mathfrak{R}(s)>0, \quad \mathfrak{R}(f)>0, \quad \mathfrak{R}\left(f^{\prime}\right)>0$, $\Re(a-c-d-e+1)>0$, and $\mathfrak{R}\left(a^{\prime}-c^{\prime}-d^{\prime}-e^{\prime}+1\right)>0$, the following result holds true:

$$
\begin{aligned}
& \left.\left.\int_{0}^{\infty} e^{-s t}\left\{\int_{0}^{t} \tau^{f-1}(t-\tau)^{f^{\prime}-1}{ }_{5} F_{5}\left[\begin{array}{c}
a, 1+\frac{1}{2} a, c, d, e \\
\frac{1}{2} a, a-c+1, a-d+1, a-e+1, f
\end{array}\right] \tau s\right] \times{ }_{5} F_{5}\left[\begin{array}{c}
a^{\prime}, 1+\frac{1}{2} a^{\prime}, c^{\prime}, d^{\prime}, e^{\prime} \\
\frac{1}{2} a^{\prime}, a^{\prime}-c^{\prime}+1, a^{\prime}-d^{\prime}+1, a^{\prime}-e^{\prime}+1, f^{\prime}
\end{array}\right](t-\tau) s\right] \mathrm{~d} \tau\right\} \mathrm{d} t \\
& =\frac{\Gamma(f) \Gamma\left(f^{\prime}\right)}{s^{f+f^{\prime}}} \Delta_{9}(a, c, d, e) \Delta_{9}\left(a^{\prime}, c^{\prime}, d^{\prime}, e^{\prime}\right) .
\end{aligned}
$$

Proof. The proofs of results (13) to (57) asserted in Theorems 1-45 are quite straight forward. For example, in order to prove result (13) asserted in Theorem 1, we proceed as follows. In the general result (9), if we get $p=q=p^{\prime}=q^{\prime}=2, a_{1}=a, a_{2}=b, \quad a_{1}^{\prime}=a^{\prime}, a_{2}^{\prime}=b^{\prime}, b_{1}=c$, $b_{2}=d, b_{1}^{\prime}=c^{\prime}, b_{2}^{\prime}=d^{\prime}, k=k^{\prime}=s, \mu=d$, and $\nu=d^{\prime}$, then it takes the following form:

$$
\begin{aligned}
& \int_{0}^{\infty} e^{-s t}\left\{\int_{0}^{t} \tau^{d-1}(t-\tau)^{d^{\prime}-1}{ }_{2} F_{2}\left[\begin{array}{l}
a, b \\
c, d
\end{array} \mid \tau s\right]{ }_{2} F_{2}\left[\begin{array}{l}
a^{\prime}, b^{\prime} \\
c^{\prime}, d^{\prime}
\end{array} \mid(t-\tau) s\right] \mathrm{d} \tau\right\} \mathrm{d} t \\
& =\Gamma(d) \Gamma\left(d^{\prime}\right) s^{-d-d^{\prime}}{ }_{2} F_{1}\left[\begin{array}{c}
a, b \\
c
\end{array}\right] 1{ }_{2} F_{1}\left[\begin{array}{c}
a^{\prime}, b^{\prime} \\
c^{\prime}
\end{array}\right] .
\end{aligned}
$$

We now observe that the twice ${ }_{2} F_{1}$ appearing on the right-hand side of (58) can be calculated by Gauss' summation theorem (3), and we easily arrive at the desired result (13). This completes the proof of result (13) asserted by Theorem 1 . The remaining results (14)-(57) can be established in a similar way. We would like to leave the details to the interested readers.

\section{Corollaries}

In this section, we shall mention several known results of our main findings:

(a) In Theorems $1,2,4,40,42$, and 44, if we take $d=b$ and $d^{\prime}=b^{\prime}$, we get the known results due to Milovanovĩ et al. (Theorems 2.1, 2, and 2.4 in [6] and Corollary 1.3 and 1.5 in [7], respectively).

(b) In Theorems 3 and 11, if we take $d=b$ and $d^{\prime}=1-a^{\prime}$, we get the known results due to Milovanovic̃ et al. (Theorems 2.3 in [6] and Corollary 4 in [7], respectively).

(c) In Theorems 5 to $8,13-16$, and 26-29, if we take $d=b$ and $d^{\prime}=c^{\prime}$, we get the known results due to Milovanovic et al. (Theorems 2.5 to $2.10,2.14$ to 2.16, $2.23,2.25$, and 2.29 in [6] respectively).

(d) In Theorems 20-23, if we take $d=1-a$ and $d^{\prime}=c^{\prime}$, we get the known results due to Milovanovic et al. (Theorems 2.11 to 2.13 and 2.27 in [6], respectively). (e) In Theorems 31-34, 36-38, 40, 41, and 43, if we take $d=c$ and $d^{\prime}=c^{\prime}$, we get the known results due to Milovanovic et al. (Theorems 2.17 to 2.22, 2.31, 2.33, 2.35, and 2.37 [6], respectively).

(f) In Theorems 9, 17, 30, 35, 39, 42, and 44, if we take $d=b$ and $f^{\prime}=c^{\prime}$, we get the known results due to Milovanovic et al. (Theorems 2.24, 2.26, 2.30, 2.32, $2.34,2.36$, and 2.38 in [6], respectively).

(g) In Theorem 24, if we take $d=1-a$ and $f^{\prime}=c$, we get the known result due to Milovanovic et al. (Theorem 2.28 in [6]).

(h) In Theorem 45, if we take $f=c$ and $f^{\prime}=c^{\prime}$, we get the known result due to Milovanovic et al. (Theorem 2.39 in [6]).

(i) In Theorem 18, if we take $d=1-a$ and $d^{\prime}=1-a^{\prime}$, we get a known result due to Milovanovic et al. (Corollary 2 in [7]).

(j) In Theorem 19, if we take $d=1-a$ and $d^{\prime}=b^{\prime}$, we get a known result due to Milovanovic et al. (Corollary 6 in [7]).

\section{Conclusion}

In this note, our aim is to demonstrate how one can easily obtain in all forty-five attractive Laplace transforms of convolution type related to the generalized hypergeometric functions from the general result recorded in [5].

Applications related to engineering will be addressed in the next study. It is hoped that the results could be of potential use in the areas of engineering mathematics and mathematical physics.

\section{Data Availability}

No data were used to support this study.

\section{Conflicts of Interest}

The authors declare that there are no conflicts of interest regarding the publication of this paper. 


\section{References}

[1] G. V. Milovanovic̃ , R. K. Parmar, and A. K. Rathie, "Certain Laplace transforms of convolution type integrals involving product of two special $F_{p}$ functions," Demonstratio Mathematica, vol. 51, pp. 264-276, 2018.

[2] G. Milovanovic, R. Parmar, and A. Rathie, "A study of generalized summation theorems for the series ${ }_{2} F_{1}$ with an applications to Laplace transforms of convolution type integrals involving Kummer's functions ${ }_{1} F_{1}$," Applicable Analysis and Discrete Mathematics, vol. 12, no. 1, pp. 257-272, 2018.

[3] E. D. Rainville, Special Functions, Macmillan, New York, 1960.

[4] H. Exton, Multiple Hypergeometric Functions and Applications, Halsted Press John Wiley \& Sons, Ellis Horwood, Chichester, New York, London, Sydney and Toronto, 1976.

[5] H. Exton, Handbook of Hypergepmetric Integrals, Theory, Applications, Tables, Computer Programs, Halsted Press John Wiley \& Sons, Ellis Horwood, Chichester, New York, Brisbane and Toronto, 1978.

[6] L. J. Slater, Generalized Hypergeometric Functions, Cambridge University Press, New York, NY, USA, 1966.

[7] L. J. Slater, Confluent Hypergeometric Functions, Cambridge University Press, New York, NY, USA, 1960.

[8] C. F. Gauss, "Disquistriones genarales area seriem infinitam," Thesis, Ges. Werke Gottingen, Gottingen, 1866.

[9] Y.-S. Kim, A. K. Rathie, and Rathie, "Applications of generalized kummer's summation theorem for the series ${ }_{2} F_{1}$," Bulletin of the Korean Mathematical Society, vol. 46, no. 6, pp. 1201-1211, 2009. 